\title{
THE USE OF ANECDOTES IN TEACHING THE INTRODUCTORY STATISTICS FOR THE PROSPECTIVE TEACHERS
}

\author{
SAnG-Gone LEe
}

\begin{abstract}
Anecdotes can produce an emotional and simple response that decreases stress and anxiety in a classroom. The use of anecdotes in building concepts of statistics can support an effective way of teaching and learning statistics. Particularly, we demonstrate several anecdotes including pictures as the medium of image that are designed to motivate statistical ideas by placing them at the beginning of a lecture and by appealing to prospective teachers weighed down. Our purpose is that under the constructivist view, prospective teachers have an opportunity effectively to teach statistical concepts using humorous anecdotes and to experience significant beliefs on identifying some frequent misconceptions in statistics. At this procedure, the anecdotal teaching practice is concerned with describing and evaluating many humorous anecdotes we have found useful in teaching introductory statistics. We hope that this paper can be helpful to prospective teachers who will teach students such topics as descriptive statistics, sampling, and hypothesis testing.
\end{abstract}

\section{Introduction}

Statistics is part of the mathematics curriculum for secondary school. This teaching usually includes the usefulness of statistics for daily life and its instrumental role in other disciplines. If most of students in a classroom have little understanding of basic statistical concepts or cannot interpret the results in a meaningful way(Vee and Khoon,1999), this difficulty generally may stem from the fact that statistics requires

Received November 4, 2009. Accepted December 7, 2009.

2000 Mathematics Subject Classification: 97U70.

Key words and phrases: Anecdote, Constructivism, Prospective teacher.

This paper was supported by research funds of Jeonbuk National University in 2009 . 
absorbing a great deal of specialized concepts used to collect data concerning a particular area of investigation and to draw conclusions in situations where uncertainty is present. Therefore, looking at one of representative examples of learning statistics, students frequently fail to connect their underlying knowledge with statistical contents such as the random variable, sampling distribution, and testing hypotheses that are abstract or counter-intuitive to them. Nonetheless, we believe that most of secondary mathematics teachers teach statistical contents in the same way they are presented in the text with the formula-based approach. As noted by Stockburger(1996) a great deal of importance is placed on how do the calculations rather than a reasonable insight into what they were doing. It is therefore important that we develop new approaches to motivate prospective teachers to pursue statistical teaching that may be most effective when the contents under consideration can be connected with their present knowledge.

We introduce anecdotes to connect underlying concepts by placing them in a class and by appealing to prospective teachers. The humorous anecdotes can have the remarkable ability to reduce stress and anxiety of prospective teachers, and thereby can improve an atmosphere conductive to learning(Knowles,1984) and communication between lecturer and prospective teachers(Talbot, 2000). Furthermore, when statistics in a classroom are presented in a humorous manner, anecdotes will make statistical concepts more interesting to prospective teachers, and increase the absorbed amount of learning, and promote long-term recall(Korobkin,1988). Therefore, they may more gradually promote attendance because statistics can not be a boring subject where prospective teachers have afraid to ask questions and solve problems more laborious. When the anecdotes are specific, related, and appropriate to a subject matter, prospective teachers will recognize a new perspective that may lead to a novel cognitive insight(Ziv, 1988).

Anecdotes can open and allow prospective teachers to interact with themselves, many of whom have divergent views and options previously suppressed by the traditional environment(Ziv,1983). The result of this interaction may provide prospective teachers with opportunities to react on their own ideas. The anecdotes will give prospective teachers a vehicle to share their thoughts with other learners. When prospective teachers actively share in the humorous anecdotes, they will cultivate free interaction and idea generation while reducing social anxiety and conformity. In contrast, although humorous anecdotes may differ from 
situation that prospective teachers would desire and create on themselves, prospective teachers will spend less time looking for answers and more time analyzing, reflecting, and developing for an understanding. Therefore, allowing prospective teachers to construct their own knowledge through the divergent thinking process will encourage them to grasp concepts quickly(Walter,1990). In the end, humorous anecdotes derived from this paper will allow prospective teachers to cognitively implement anecdotes in two ways; First, they can represent abstract concepts in terms of concrete ideas which fall within their experiences that transform abstract concepts into anecdotes under lecturer's guiding. Second, they can make a classroom more enjoyable, reduce anxiety, and improve the learning environment.

In the future teaching, anecdotes will create a powerful cognitive tool to motive new statistical ideas based on appeal and effectiveness if prospective teachers use suitable anecdotes. It is anticipated that humorous anecdotes can map their statistical classroom to a more effective environment that connects concrete concepts and enhances communication to establish a warm relationship between prospective teachers and their students.

Although several studies(Friedman, Halpern and Salb, 1999) have shown that humorous anecdotes can increase the advantages mentioned above, prospective teachers will need more time and practice to produce a wide variety of humorous anecdotes, selecting only those appropriate for the class, and using the anecdotes so it is not to hurt the feelings of concepts. Moreover, most reports with the research on humor and learning have not been demonstrated to improve when a teaching includes humorous anecdotes(Gruner, 1967). No study on humor has exactly addressed the method of evaluating learning the researchers used(Kaplan and Pascoe, 1977). In this paper, we discuss that the anecdotes can be used to bring statistical concepts into focus using them within a point of constructivist view, and present pictures describing illustrations in conjunction with them that help prospective teachers to understand concepts of statistics and to clear up some frequent misconceptions in statistics. In this process, we consider anecdotes with pictures, some well known and some new, that we have found beneficial. We would like to characterize this paper as creating " a stimulus of new ideas, an improvement of learning environment, and an intuition to concepts from image constructions". 


\section{Characteristics and Theoretical Views in Teaching Sta- tistics with Anecdotes}

According to Plato's dialogue, Socrates attempted to demonstrate how a mathematically uneducated slave solves a geometrical problem. Eventually, Socrates concluded that even if the learning is only the recovery of the pre-existent knowledge in the human soul, the answer to a problem can be transferred from a lecturer to a learner by guiding the learner to find out for himself. Through this experiment, we can find that the dialogue with anecdotes can serve as a controlled guidance for learners whenever constructing anecdotes to explain key concepts in the introductory statistics.

In ancient Babylonia, the Talmud(Shabbos 30b) recorded that as a tool to lively present an ethical and religious instruction, Rabbah states something humorous before starting a lecture, laughs together, and then begins the main lecture(Sacks,2008). Considering the Talmudic sages' humorous lecture, humorous anecdotes have been naturally shown to have both psychological and physiological effects on learners. Psychological function of humorous anecdotes produces a cognitive shift that allows learners to distance themselves from the complicated situation. It promotes an objectivity that buffers the negative feelings and provides a sense of stimulation that can improve learner's mental performance. Unfortunately, humorous things seem to be such a complex human phenomenon that it cannot be easily analyzed and fully explained by researchers(Ziv, 1976).

Though anecdotes permit learners to deal more effectively with an unpleasant situation, its accompanying physiological response is usually laughter in some well known form. While the laughter translates into physiological effects throughout the entire body, the psychological effects reduce physical stress. Thus, it can be hypothesized that the laughter following anecdotes has a releasing effect on the process of emotional response. Psychologically, we can expect that humor and laughter can reduce anxiety, help stress relieve, and increase mental sharpness (Cousins,1991). Moreover, the laughter tends to open learners to divergent thinking previously suppressed by the traditional environment. Glenn(2002) suggests that the humor can help learners engage in the learning by creating a positive emotional and social environment which learners may more and more absorb the information being presented. Therefore, humorous anecdotes make it easier for learners to work cooperatively and learn from each other as well as 
from the lecturer(Hashem,1994). The humorous anecdotes can serve as a bridge(Pollio \& Humphreys,1996) between a lecturer and a learner by demonstrating a shared understanding. Therefore, using humorous anecdotes in a positive way may help to close the gaps between a lecturer and a learner while stimulating cooperative work.

Collectively, anecdotes are very short stories that can add impact to our lecture and are effective in reducing learner's stress while motivating a learning activity. Therefore, as a pedagogical tool of teaching statistics using the humorous anecdotes in a classroom, we obviously can anticipate the following characteristics of the anecdotes to learners.

It will increase:

- Motivation

- Absorption

- Attendance

- Positive attitude

- Lecturer-learner communication

- Individual and group cooperation work

- Divergent thinking

- Long-term recall

while decreasing:

- Anxiety

- Stress

In past centuries, constructivism in learning is based on the theory that knowledge is not transferred from one lecturer to another. In this theory, it is important that learners are engaged with the subject materials to grasp concepts for themselves rather than holding their hands, so that they can relate concepts to real application and construct their own knowledge in a passive manner. In this paper, we would like to adopt the constructivist thinking of teaching statistics with anecdotes for a theoretical framework that allows prospective teachers a better performance as secondary teachers.

The following of this chapter presents that teaching the introductory statistics using anecdotes includes effective characteristics on the basis of constructivist views. In the act of constructing concepts, we can observe carefully five attitudes: First, a concept which is illustrated in a humorous manner might be learned more easily than a concept presented in a formula style. Therefore, humorous anecdotes will heighten interest in our teaching. Statistical concepts are lightened and made more approachable. By lectures with humorous anecdotes illustrating concepts, prospective teachers will have motivating effects for more active and 
reflective construction. Second, humorous anecdotes may require different problem solving methods and processes than traditional activities. During the self-discovery process constructed in a little performance at the beginning of a class or a section, humorous anecdotes can actively involve prospective teachers by guiding them to find out for themselves in order to foster discussion of different solutions and strategies of solution, because the humorous anecdotes are no more or less rigorous than a straight environment in the classroom. Third, humorous anecdotes tends to open prospective teachers to divergent thinking because there is more time and energy left to learn and create by means of the reduction of stress and anxiety through laughter. Fourth, humorous anecdotes in a classroom are effective at reducing stress, facilitating understanding, and encouraging participation. The humorous anecdotes help to close the gaps between prospective teachers stimulating cooperative work. Therefore, prospective teachers can work in groups of many different ways, many of which are based on acquisition of knowledge. The anecdotes may help them become aware of the relevancy and meaningfulness of what they are learning. Finally, all lecturers will have less success if lecturers simply feed learners their knowledge with no beliefs about the importance of teaching statistics using anecdotes.

\section{Strategies to Produce Humorous Anecdotes in the Class- room}

How can we cultivate our own sense of humor? Even if our humorous anecdote is accepted as an effective tool, how can we put this anecdote into action in a classroom? These problems are faced when lecturers teach statistics using anecdotes in a class. No book provides step-bystep guidelines on how to create a humorous anecdote appropriately. For example, we can only find that each humorous forms[Table 2] is classified according to a variety of humorous sources[Table 1](Wandersee, 1982). It goes without saying that many lecturers fear to let a sense of humor come through to learners, because the front of the classroom is already making afraid. Nevertheless, reducing anxiety about presentation skills will support an even chance to develop self-confidence, shared humorous anecdotes, and timing. We believe that production of an anecdote can be presented with a gradual and behavioral process in which lecturers describe their ideal feelings and accept rather than judge. Moreover, the text style of anecdotes is not for everyone, but a picture an be useful in helping learners to remember a concept about abstract content. 
Because images are impressed upon the mind more easily and are retained longer, presenting humorous anecdotes with a picture may serve as demonstrable cues for recalling concepts. But, attention will be focused on teaching statistics using the humorous anecdotes, which may distract learners from a lecture presented in a straightforward manner.

Integration of humorous activities and comments into an instructional sequence may be a slow and cautious process that requires a lot of trials and revision. Lecturers' teaching should always evolve into an activity that promotes learners' thinking, discussion, and motivation. Before teaching, lecturers need to examine the subject matter, their own personal brand of humor, their presentation skills, and their needs in order to develop anecdotes. Thoughtfully designed humorous anecdotes and presentation skills only can be used as a strategy in activity development. While the intended anecdote does not significantly reduce stress and anxiety, appropriate use has a positive impact on the learners.

When lecturers teach statistics using humorous anecdotes, the first thing that they can do for learners is to have learners feel relaxed in the classroom. Next, they must know the reason why lecturers use anecdotes and how to use anecdotes effectively. Finally, they have to notice that it depends on the nature of contents whether a humorous anecdote and its picture should be inserted or not at the beginning of the lecture. Therefore, anecdotes are very sensitive, so great care should be considered in teaching statistics using them. As useful strategies that have been considered to create anecdotes in the classroom, we would like to suggest the followings for heightening the memorable teaching of humorous anecdotes with pictures:

- Choose an effective time for anecdotes

- Notice the first day of class unfamiliar with learners

- Do not make an anecdote with an unusual meaning

- Use plenty of graphics and pictures in Web sites

- Use anecdotes related concepts for recalling anecdotes

- Integrate humorous activities and comments into the learning objective

- Make smooth progress in a lecturer's speech to speaking anecdotes

- Revise anecdotes of another person into a lecturer's own style

- Keep quiet if need arise in the classroom 


\section{The Use of Anecdotes with Pictures in Teaching Statistics}

Many times the resources may be available for a census, but the nature of the population requires a sample. We usually try to do experiments for a great deal of statistical information. After a series of experiments from the population, sample data are collected and analyzed in terms of various techniques for descriptive statistics. Then, inferential statistics determines whether the observed differences in the sample statistics happen to have variation as the cause of the difference. Eventually, inferential statistics permits us to draw conclusions concerning the effects of experimental variables and to generalize these conclusions to the population.

Anecdotes with pictures may be more effective in conveying abstractedly statistical concepts to learners that we can't simply quote the underlying knowledge without presenting the images and in decreasing some common misconceptions in statistics. When properly used, if prospective teachers are discouraged by negative expectations of traditional statistics lecture, humorous anecdotes with pictures may help to motivate prospective teachers with a wide variety of mathematical backgrounds, encourage classroom discussions by stimulating the interest of prospective teachers, elicit multiple responses from prospective teachers, and demonstrate the relevance of statistics to everyday life.

We would like to practice our lecture before asking prospective teachers to engage the class as a whole in discussion about main concepts they will be learning. When a lecture is given, we suggest that first, lecturers must comment anecdotes appropriate to the medium by means of which they can communicate with prospective teachers. Second, a picture explaining various features by moving their hands must be placed on the overhead projector. But, all comments of the lecturer can not necessarily coincide with the meaning the lecturer intended to convey. Third, the humorous anecdotes must have been inserted at the beginning of the lecture and proceeded at two or five minutes into the lecture, depending on the concepts. Finally, by applying suitable strategies to produce the humorous anecdotes, the teaching practices using them under the constructivist view will support a source of information starting with which they organize concepts having meaning, and reconstruct abstract concepts which serve as an intuitive concept free from common misconception. At the end of the class, lecturers may even change to a more effective environment to facilitate teaching of statistical concepts using simulations and animations of computers. As a result, the use of 
suitable, specific anecdotes can provide prospective teachers with a new perspective on the introductory statistics.

In this chapter, what we mean by teaching statistics with anecdotes suggests the teaching practices that brings cohesion to anecdotes and pictures, showing when, how, and why to use them in various situations including some misconceptions in statistics. Moreover, for prospective teachers to have an opportunity on experiencing significant changes of teaching statistics, we are ourselves constructing the appropriate pictures to teach statistics with anecdotes under the constructivist ideas. We demonstrated examples to attempt teaching statistics using anecdotes with pictures as following three steps: (1)introduce an anecdote, (2)show a picture transferring abstract concepts to the intuitive image, and (3)summarize or illustrate statistical concepts to focus prospective teachers on pictures presented as the medium of image and to dispel them from common misconception in statistics.

Let us start to show the first anecdote that demonstrates a distorted view in the advertising statement that follows after the growth of the misleading statistic.

Anecdote 1. One gas station displayed in large letters, "The lowest price of Gas in this Street." The neighbor boasted, "The lowest price of Gas in the town." A third on the next block claimed, "The lowest price of Gas in Seoul." A fourth boldly stated, "Sell Gas for the lowest price in Korea."

Present an Anecdote 1:

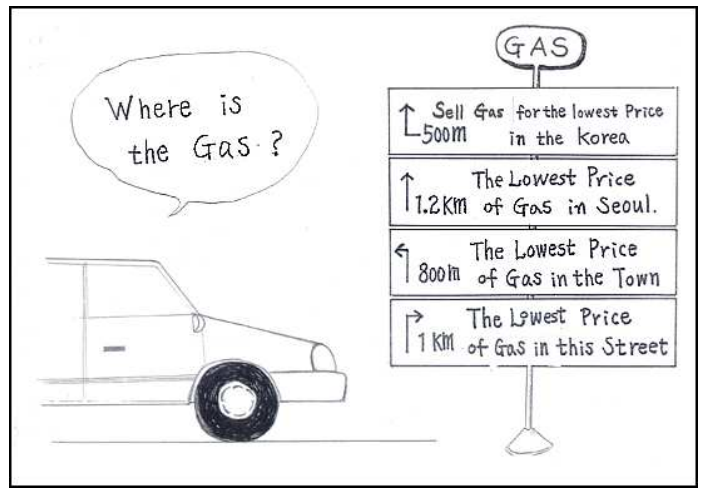

Figure 1. Image of Anecdote 1

Statistical Treatment: Statistical errors abound in our newspapers, 
magazines, web sites, and advertisements. Some errors come up with statements that are totally meaningless not only because the scales properly are unmarked but also because the more subtle interpretations are unfolded even though widely believed.

\section{A. Descriptive Statistics}

To organize data without obscuring the essential information we need a histogram accomplished by grouping arrayed data into small number of classes. A graphical display of data in a histogram is more likely to present relations that may be overlooked in a chart. But, some prospective teachers may claim it burdensome to construct such statistical description.

Anecdote 2. A manager in a large discount store draws a bar graph representing the weekly dollar volume for four sales associates working under him, but he stretches $Y$-axis and contracts $X$-axis in the bar graph. His purpose uses this figure to dismiss Mr.D for neglect of duty, whereas Mr.D has not been caught off guard and has been taking prolonged coffee breaks each morning. What does the manager do?

Present an Anecdote 2:

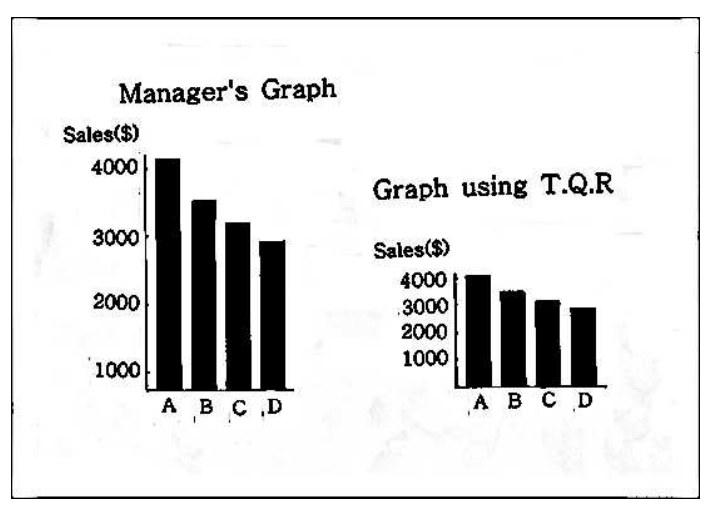

Figure 2. Image of Anecdote 2

Statistical Treatment: A manager has to draw the bar graph for four sales associates applying the three-quarters rule(T.Q.R) that the height of the maximum point of $\mathrm{Y}$-axis is approximately equal to three-quarters of the length of $\mathrm{X}$-axis.

Anecdote 3. On one day of last fall, a farmer at Jeju island picked 
oranges from trees selected randomly. He went to the Nong-Hyup market which an orange grading machine works to decide the grading level by the size of oranges and packs them in the same box. Looking at the number of oranges in each box, the farmer recognized that if the resulting grading is too fine to be commercially useful, customers may be confused by only several choices and may be dissatisfied when they buy a certain orange box. The farmer changed his mind to check whether oranges of very different sizes are graded as equivalent.

\section{Present an Anecdote 3:}

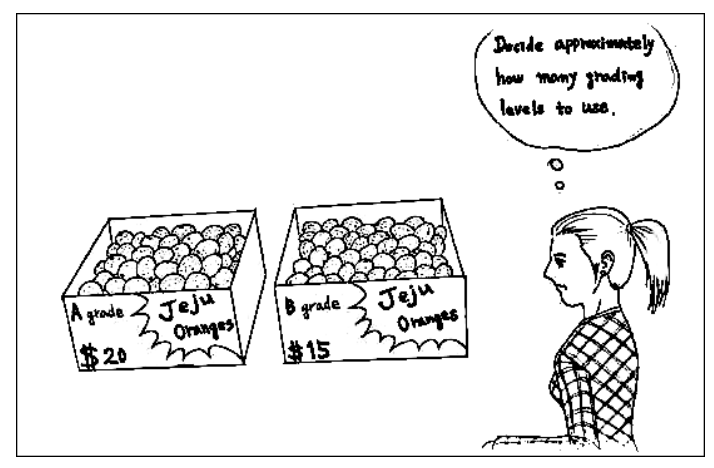

Figure 3. Image of Anecdote 3

Statistical Treatment: A histogram is a bar chart of a frequency distribution: Data categories are displayed on $\mathrm{X}$-axis, and rectangles are displayed on $\mathrm{Y}$-axis. Height of a rectangle $=$ (Relative frequency of a class)/(Width of a class interval). The number of classes depends on such factors as the number of observations, the purpose for which the distribution is being prepared, and the arbitrary preferences of the analyst(Sanders \& Murph,1976).

The term average is used to describe some specific property. That is the reason why there are many kinds of average. Some are better suited to certain situations than others. The three averages that we frequently use are the mean, median, and mode. The other particular averages that we use less frequently are the geometric mean and harmonic mean. Anecdote 4. A person who can have his head in a cooking range and his feet in a refrigerator says that he feels comfortable on the ground with his average temperature. Who can say so?

Present an Anecdote 4: 


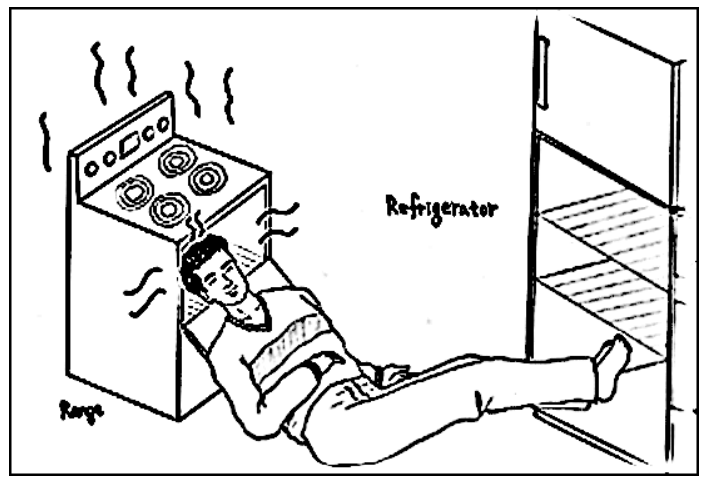

FiguRE 4. Image of Anecdote 4

Statistical Treatment: The sample mean of $n$ measurements $x_{1}, x_{2}, \cdots, x_{n}$ is the sum of these measurements divided by $n: \bar{X}=\sum_{i=1}^{n} x_{i} / n$. Though the mean is the most commonly used average, it is too greatly influenced by outliers to be representative of all. Of course, the mean to more complicated distributions, including continuous distribution, is more delicate.

Sometimes dispersion is more important than average. Formal measures to express dispersion include the range, mean deviation, standard deviation, variance, interquartile range, quartile deviation, and so forth. Anecdote 5. During the Korean War in the 1950's, a lieutenant and his troops who were backing to the south region from Seoul discovered that there were no boats available for the crossing when they have just arrived at a small river. The lieutenant conjecturing that the average depth at this time of year was less than one meter, gave the order to cross on foot. After the crossing, the lieutenant discovered that many soldiers had drowned. Why did the event happen?

Present an Anecdote 5:

Statistical Treatment: While the mean is a representative value about which the measurements are distributed, the variance describes the dispersion of the individual measurements about its mean.

\section{B. Inferential Statistics}

Sampling occurs frequently in daily events and provides sufficient information as a basis for inferences about the population. If we make an inference about a population from samples, we may use the sample results as a representative of the population. Unfortunately, we cannot 


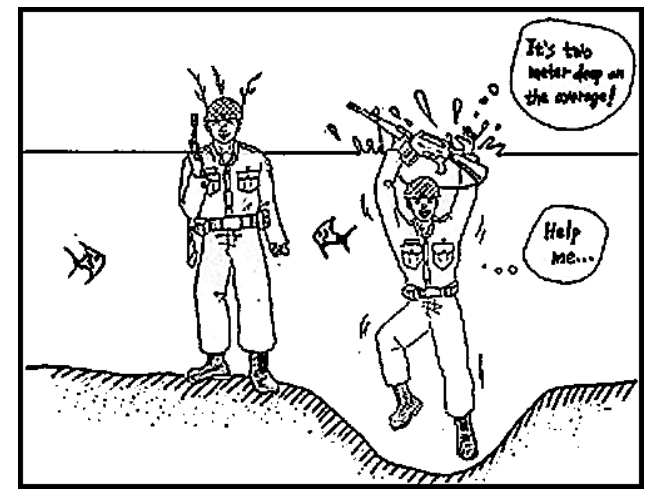

Figure 5. Image of Anecdote 5

expect a sample result to have exactly the same value as some population characteristics, because sampling error exists. Consequently, we should allow sampling as efficient manner in terms of minimizing the sampling error. Here is an activity that we can use many times to introduce students to the idea of the way in which a sample is selected or the way in which the data is collected from the sample.

Anecdote 6. When sample candies from a population of a certain brand of candy are repeatedly drawn, each sample of size 48 is known with containing 12 red candies. A lecturer give all students one certain brand of candy as soon as the class starts. When students open their own bag, a lecturer instructs students to count the numbers of each color and find the total number of candies in the bag. Ask a question whether how many "red devils" are in the bag.

\section{Present an Anecdote 6:}

Statistical Treatment: The probability assigned to an event as a numerical value to measure how likely it is accessible suggests that the event may take place when the experiment is repeated.

Anecdote 7. A salaried man who had a telephone directory alphabetized by types of business wanted to carry out a survey of the fixed place where people went on their summer vacation. He randomly opened his telephone directory and called up an arbitrary number of the page. "Excuse me, would you please tell me where you spent the vacation this summer?" Back came the answer, "Mt.Seorak". He called the next number, and again, "Mt.Jiri". The third number was "Mt.Seorak", while the next five were all "Mt.Halla". But, after finishing all phone-calls, he realized 


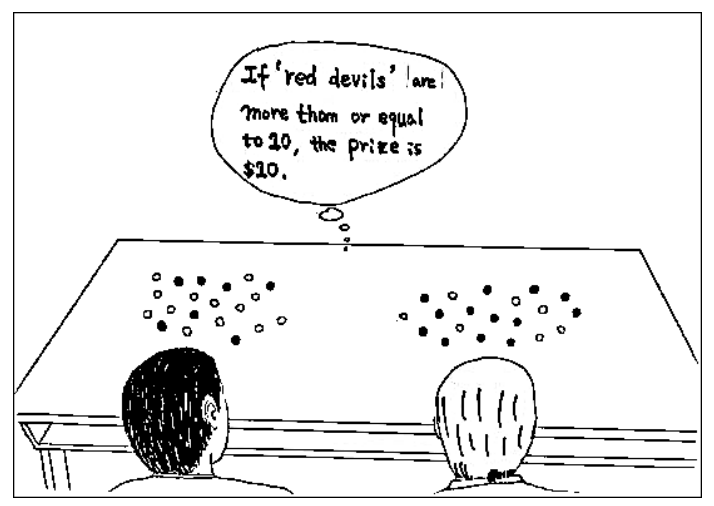

Figure 6. Image of Anecdote 6

that the directory was opened at the Alpine club. What had happened? Present an Anecdote 7:

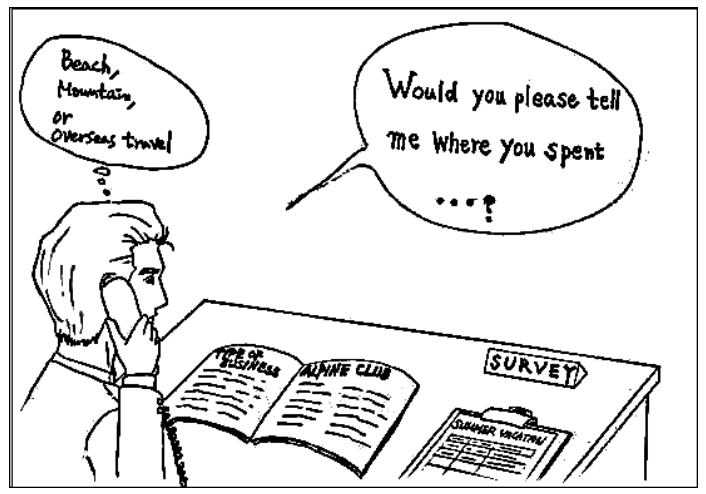

Figure 7. Image of Anecdote 7

Statistical Treatment: A random sample of size $\mathrm{n}$ is a sample that is selected from a population in a way that each element of size $\mathrm{n}$ is equally likely to be chosen.

Anecdote 8. A reporter says that LSG Telecom was ordered by the Fair Trade Commission(FTC) to stop advertising that "twice as many customers use LSG Telecom for their cellular phone as the other Telecom." The claim of LSG Telecom is based on a survey of 50,000 randomly selected individuals from a list of 5,000,000 subscribers to mobile communication. LSG Telecom received 8,279 responses, with 2,591 saying 
that individuals used $L S G$ Telecom and only 1,089 reporting that they used the other Telecom. Why was LSG Telecom ordered by the FTC to stop such advertisement?

\section{Present an Anecdote 8:}

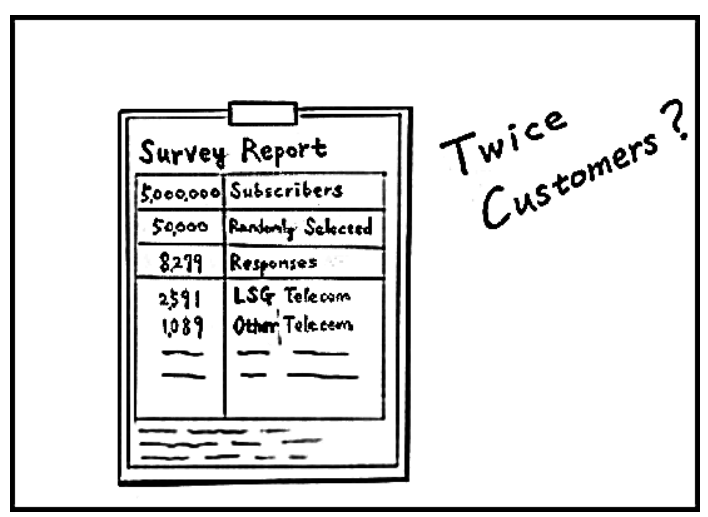

Figure 8. Image of Anecdote 8

Statistical Treatment: One sample should have been randomly sampled from each of randomly selected ones to obtain a representative sample. Because people who respond to surveys are very interested in the subject of the questionnaire, and people with little interest in the subject often choose not to respond, it is not the number of responses that matter but the rate of response.

Anecdote 9. Two people were hired as interviewers for the $A B C$ University to look at the cheating problem on campus. Since two surveyors asked students questionnaires premised on anonymity, more students were likely to admit cheating on their final examination to peers than to elderly people in a conservative suit. Finally, the ABC University found that about $20 \%$ students have confessed to cheating within the last semester. What issues are impressed to increasing responses in the survey?

\section{Present an Anecdote 9:}

Statistical Treatment: The interviewing method can affect responses: The age, sex, clothing, vocal intonations, and manner of the interviewer are becoming an effective factor in a survey. In fact, the higher rate of response a sample has, the higher validity of survey we can have in our testing. 


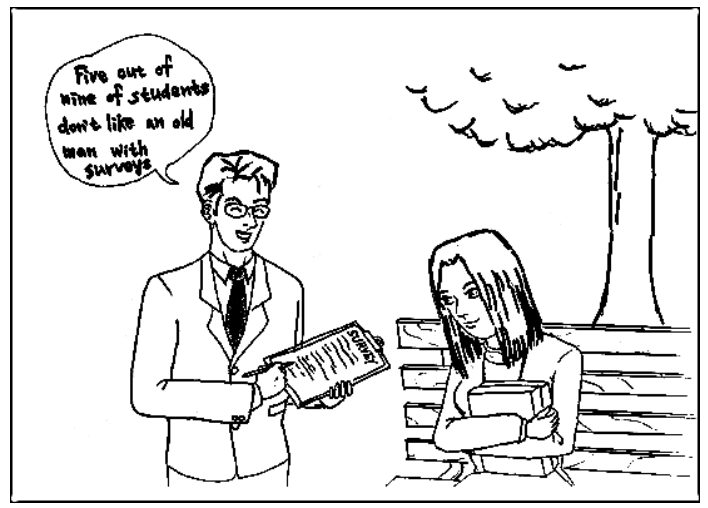

Figure 9. Image of Anecdote 9

Anecdote 10. Nowadays, the unsteady occurrence of Gas-value frequently increases. An elderly man has to make up his mind about the problem of deciding whether to purchase an old hybrid car at its asking price. Will he buy the vehicle at its asking price or not?

Present an Anecdote 10:

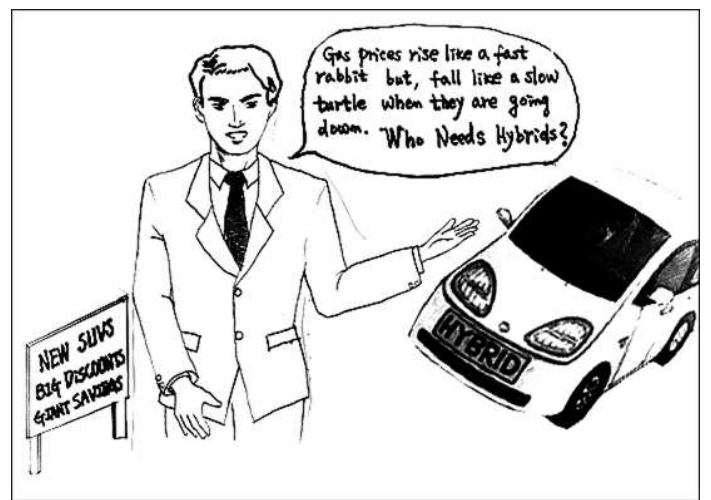

Figure 10. Image of Anecdote 10

Statistical Treatment: The first step in hypothesis testing is to suppose that the null hypothesis $H_{0}$ corresponds with the assumption that the value of the vehicle is equal to the asking price, and the alternative hypothesis $H_{1}$ is that the vehicle is worth less than its asking price. After that, he can determine a distribution applicable in hypothesis testing, calculate sample statistics, compare the critical ratio with the stated 
decision rule, and draw a statistical conclusion concerning the null hypothesis.

\section{Conclusion}

Prospective teachers have identified statistics as one of difficult courses in university(Gardner and Hudson, 1999). If teaching the introductory statistics includes humorous anecdotes, we believe that prospective teachers can be closer to understanding key concepts by constructing their own knowledge in a passive manner under lecturer's guiding. But, because humorous anecdotes may be highly personal, subjective, and contextual, lecturers cannot always predict the way that they will be received. Therefore, lecturers always need to minimize distraction from the learning that is dangerous in unrestrained anecdotal use. Whenever lecturers teach statistics with humorous anecdotes, it's the most reasonable way that they should have at least a chance academically to insure whether the insert can be considered to be humorous, appropriated in content, and related to concepts being covered.

As mentioned in this paper, humorous anecdotes are surely a powerful instructional tool for teaching, and may create a positive atmosphere, reduce stress, enhance recall, and build communication between lecturers and learners. Especially, when lecturers teach the introductory statistics at the beginning of a class or a section, humorous anecdotes can act as more productive tool by illustrating a picture including an anecdote on the overhead projector (or on the presented screen) and using useful strategies to produce them. We hope that this paper will be helpful to prospective teachers who may have immediate applications and needs using anecdotes.

\section{References}

[1] N. Cousins. Anatomy of an illness as Perceived by the Patient. N.Y. Bantam, 1991.

[2] J. Flowers. The Value of Humor in Technology Education. Technology Teacher, 60:10-13, 2001.

[3] P. L. Gardner and I. Hudson. University Students's Ability to Apply Statistical Procedures. Journal of Statistics Education, 7, 1999.

[4] R. Glenn. Brain Research: Practical Applications for the Classroom. Teaching for Excellence, 21:1-2, 2002.

[5] C. R. Gruner. The Effect of Humor on Speaker Ethos and Audience Information Gain. Journal of Communication, 17:228-233, 1967. 
[6] N. Halpern H. Friedman and D. Salb. Teaching Statistics using Humorous Anecdotes. Mathematics Teacher, 92:305-308, 1999.

[7] M. E. Hashem. Play and Humor in the College Classroom: Using play as a Teaching Technique in Interpersonal Communications. Paper Presented at the Annual Meeting of the Central States Communication Association, Oklahoma City, 1994. ERIC Document Reproduction Service No.ED 372442.

[8] R. M. Kaplan and G. C. Pascoe. Humorous Lectures and Humorous Examples: Some Effects upon Comprehension and Retention. Journal of Educational Psychology, 69:61-65, 1997.

[9] M. Knowles. Andragogy in Action. San Francisco; Jossey-Bass, 1984.

[10] D. Korobkin. Humor in the Classroom: Consideration and Strategies? College Teaching, 36:154-158, 1988.

[11] M. Nicaise and D. Barnes. The Union of Technology, Constructivism, and Teacher Education. Journal of Teacher Education, 47:205-211, 1996.

[12] H. Pollio and W. Humphreys. What Award-winning Lecturers say about Their Teaching: It's all about Connection. College Teaching, 44:101-106, 1996.

[13] R. P. Runyon. Winning with Statistics: A Painless First Look at Numbers, Ratios, Percentages, Means, and Inference. Addison Wesley Pub. Co, 1977.

[14] R. P. Runyon and A. Haber. Fundamentals of Behavioral Statistics 3rd ed. Addison Wesley Pub. Co, 1976.

[15] D. Sacks. What the Butterfly knows. [Online], 2008. (http://www.613.org/sacksarticle.htm).

[16] D. H. Sanders and A. F. Murph. Statistics A Fresh Approach. McGraw-Hill, 1976.

[17] D. W. Stockburger. Introductory Statistics. http://www.psychstat.missouristate.edu, 1966.

[18] M. Talbot. The Placebo Prescription. New York Times Magazine, 9:34-60, 2000.

[19] M. N. Vee and Y. W. Khoon. Using Simulation on the Internet to Teach Statistics. Mathematics Teacher, 8:729-733, 1999.

[20] G. Walter. Laugh, Teacher, Laugh! Education Digest, 55:43-44, 1990.

[21] J. H. Wandersee. Humor as a Teaching Strategy. The American Biology Teacher, 44:212-215, 1982.

[22] A. Ziv. Facilitating Effects of Humor on Creativity. Journal of Educational Psychology, 68:318-322, 1976.

[23] A. Ziv. The Influence of Humorous Atmosphere on Divergent Thinking. Contemporary Educational Psychology, 8:68-75, 1983.

[24] A. Ziv. Teaching and Learning with Humor: Experiment and Replications. Contemporary Educational Psychology, 6:37-44, 1988.

Department of Mathematics Education

Jeonbuk National University

JeonJu, 561-756 Korea

E-mail: sgl@jbnu.ac.kr 